\title{
STUDY OF A WRECK IN FORESHORE CONTEXT
}

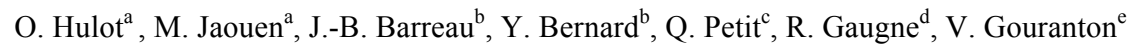

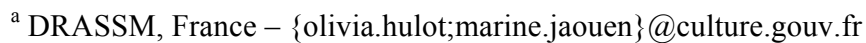 \\ ${ }^{\mathrm{b}}$ CNRS, CReAAH UMR 6566, France - \{jean-baptiste.barreau;yann.bernard $\} @$ univ-rennes1.fr \\ ${ }^{\mathrm{c}}$ CNRS, IRISA UMR 6074, France - quentin.petit@irisa.fr \\ ${ }^{\mathrm{d}}$ Université de Rennes 1, IRISA UMR 6074, France - ronan.gaugne@irisa.fr \\ ${ }^{\mathrm{e}}$ Insa de Rennes, IRISA UMR 6074, France - valerie.gouranton@irisa.fr
}

\section{Commission V}

KEY WORDS: Foreshore archaeology, Photogrammetry, Laser scanning, 3D digitization, Virtual Reality

\begin{abstract}
:
We present the study of a wreck, in a foreshore area, in the North of Brittany, France, using two different digitization methods, photogrammetry and laser scanning. The digitization process had to deal with the tide constraints. The 3D data produced using these technologies has been deployed in a large immersive infrastructure dedicated to virtual reality research, in order to propose new practises for archaeologists.

The overall purpose of our research project is to define an innovative and efficient methodology for the study and preservation of cultural heritage in an inter-tidal context. In the inter-tidal context, heritage is really fragile and the risk of destruction is real (storms, erosion, coastal development...). The traditional methods are no longer efficient. This paper describes preliminary results, through the joint work of a research institute specialized in underwater archaeology, a research laboratory of archaeology and archaeosciences, and a research laboratory in computer science.
\end{abstract}

\section{INTRODUCTION}

Alongside the study of wrecks located at great depths, another coastal area, the foreshore, has specific problems. This maritime area faces other constraints that are alternating daily tides, silting or de-silting of beaches, and development works. Archaeological foreshore sites are mostly of them submitted to the tides. Thus they cover and uncover four times a day. This constraint requires scientific teams to rethink the usual working methods for underwater or land environments. At each rising tide, the working clearance of a site is partly annihilated and must be performed again.

Foreshore archaeology is yet identified as a specific activity, requiring adapted processes. We can cite works in Australia (Anderson et al. 2012), and United Kingdom (Bell et al. 2000, Draper 2007, Nayling and Jones 2009) illustrating foreshore wreck studies. All these works highlight the problematic of the preservation of these cultural heritage sites submitted to an aggressive environment. In the Atlantic and Channel intertidal zones, the number of wrecks officially declared or simply known is now large enough to initiate thinking about a methodical, concrete and effective approach in DRASSM. Some archaeological evaluations tried to characterize some of these deposits, but they mostly remain small scale and few wrecks were the subjects of an exhaustive search. It is now necessary to develop a specific methodology, to the study these sites on the basis of effective technological tools to document the remains in a short period of time while overcoming maritime constraints.

Uncovering of the wreck «Erquy les Hopitaux» (Figure 1) Côtes-d'Armor, Brittany, France, leading to its discovery in 2002 (Rieth 2014) could be due to riprap works conducted by the French departmental direction of equipment on a nearby beach. This kind of development is common on our shores.
Sometimes they directly destruct sites and sometimes they not affect them directly, but can reveal them or accelerating their degradation, even their disappearance when they are exposed to the swell and daily tides. During winters 2013 and 2014 on the French coasts, in Charente-Maritime and in Brittany, two wrecks from 17 th century to 18 th century were dislocated within a few days during winter storms that demonstrates the urgency of the consideration of these remains particularly vulnerable to destruction.

For this campaign, we chose to assess two digitization technologies, photogrammetry (Prins and Adams 2012, Matsumoto and Ono 2009) and laser scanning (Vozikis et al. 2004, Barreau et al., 2014), and to propose a virtual reality application for immersive 1:1 study of the 3D data.

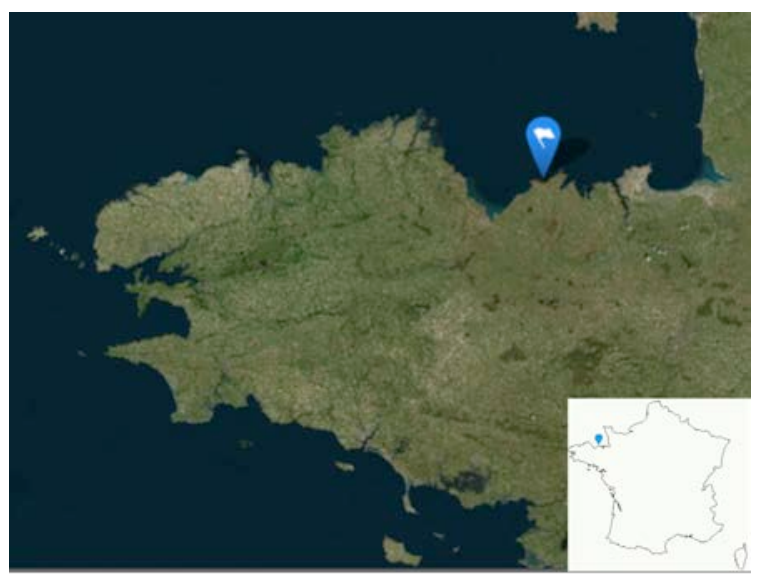

Figure 1. Erquy Les Hôpitaux, wreck position 


\section{CONTEXT OF WORK}

\subsection{Site description}

The wreck called «les Hôpitaux » (Figure 2) was discovered on the beach «Les Moutiers » in May 2002. It was assessed by the Drassm in August 2002 (L'Hour and Veyrat 2002).

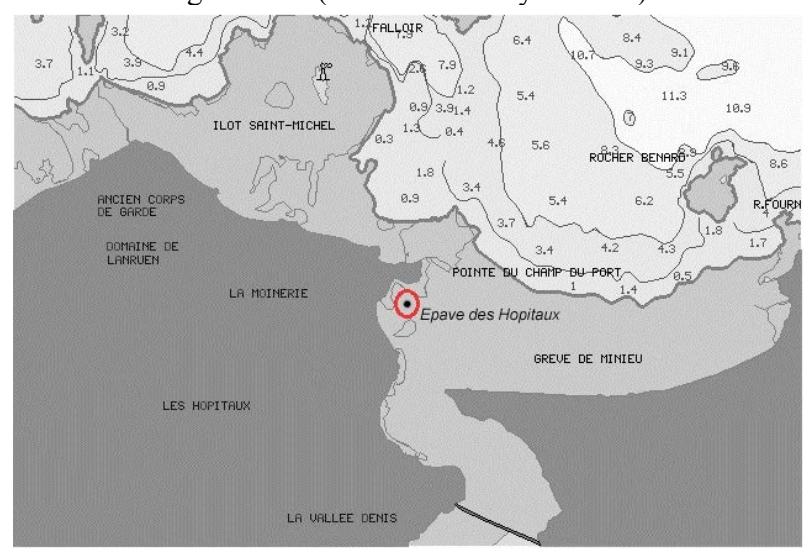

Figure 2. Site map with wreck position

The remains of the hull are located midway between the high tide and the low tide. Protected on the North by la Pointe du Champ du Port, the ship's position seems to indicate the desire to have sought shelter on the beach. Traces of fire on the starboard side let presume the cause of the loss of the vessel.

In October 2014, the site was protected by 60 to $80 \mathrm{~cm}$ of sand. To ensure maximum working time between two high tides, the archaeological dig (Figure 3) took place during a week in which the coefficients were over than 90. In fact, we know now, on the Moutier beach, the position of the wreck and the beach configuration does not require such precaution because the available working time is not significant with greater tidal coefficients.

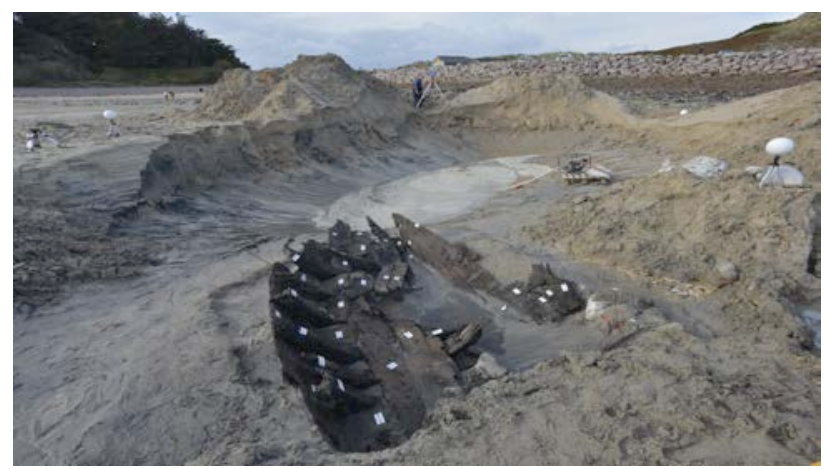

Figure 3. The wreck "Les Hôpitaux" (F.Osada/Drassm)

\subsection{Description of the wreck and archaeological interpretation}

When discovered in 2002, the wreck is characterized by two alignments of frames that join the front and rear of the vessel, drawing the outline of a small-sized ship (Figure 4). A hull is retained over 9 meters long and 3 meters wide.

The 2002 assessment and archaeological dig in 2014 helped to clarify the characteristics of the wreck.

The larboard side retains 6 frames in place ( 7 at all) and a series of 7 shell chocks. Two batten boards are also present on the frames on larboard. The larboard side consists of 5 shell platings which are assembled by means of treenails. Two barrel staves were placed against the frames and still had lime debris, indicating some evidences of the cargo. Many traces of fire, previously noticed in 2002 , have proved central to the wreck and on starboard. It reflects a damage of the ship maybe an attack that generated an outbreak of fire. This could be a voluntary fire in order to not offer the ship to the enemy.

The architecture's analysis of the boat revealed, despite of the size, that it is a robust construction, to support heavy cargo.

Small tonnage's wreck probably corresponds to a coastal boat carrying lime and even a possible cargo of slates. The naval architecture of the boat is built with elm. It is a real difficulty to obtain a dendrochronological dating with this specific wood specie. Thus, a dendrochronology analysis based on barrels staves was realised in 2002 by le CEDRE and proposed a terminus post quem in 1681 and more probably (with an evaluation of sapwood and heartwood withdrawn) around 1700 . In this period, port activity consisted mainly in coasting trade, especially with the port of Saint-Malo, situated at 20 east nautical miles of Erquy (Le Bouedec 2008). The harbour Erquy mainly exported wheat produced in the hinterland but welcomed a more diverse cargo (Le Gall and Blouin 1994). Most of the traffic (flaxseed, iron, slate, beer and lime transported in barrels) was provided by small buildings of 20 to 40 tons, through regular rotations with Saint-Malo.

In the eighteenth century the Hôpitaux's harbour was situated on an ancient area stranding and was more accessible than the main harbour of Erquy. It was protect against west winds. On a dock of stones, goods was embarked or disembarked from embarkations.

In the eighteenth century the English forces harass the French coasts many times. An eventuality is that the boat partially burnt because of a naval battle with the enemy led the crew of the ship to run aground on the coast

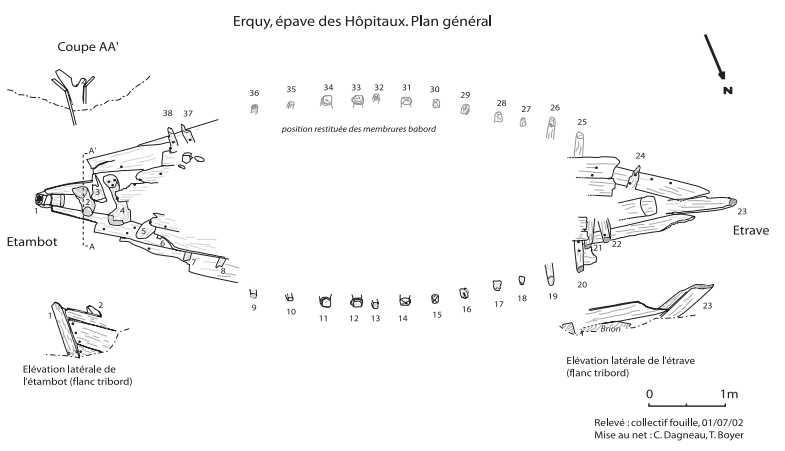

Figure 4. Sketch of the wreck

\section{WORK DESCRIPTION}

In 2014, the Drassm decided to start triennial program (conducted by O. Hulot) for study foreshore's wrecks. This project aims to search and use scientific and technical solutions for define and assess different approaches to excavate and to collect data, in particular digital data.

Because of their dimension and chronology, the first site selected is the wreck « les Hôpitaux ».

\subsection{Aims and protocol of the study}

To respect and satisfy to archaeological requirements while considering tidal constraints, the project aims to define news approaches for excavate and collect scientific data, in particular 
digital data. This project aims to combine and compare several approaches to obtain scientific data.

Protocol of the wreck 's study:

- $\quad$ Excavation of the wreck in foreshore zone

Archaeological "traditional" work: measurements and drawing

Laser and photo scanning of the wreck, with 3D rendering of the naval architecture,

Dendrochronological and xylological samples,

Taking complementary samples (caulking, lest, cargo),

Artefacts treatment (collection, inventory, management conservation...)

Site protection

Heritage development from data constituted of documentary research (historical and archival) and 3D restitution.

We focus in this work on the digitization process of the wreck and its 1:1 restitution in an immersive facility. The wreck was digitized through both laser scanning and photogrammetry. The point cloud obtained and the resulting computed mesh were displayed in a large immersive infrastructure, the Immersia facility, in order to provide a valuable working tool for archaeologists of the Drassm.

\subsection{Organisation of the on-site work}

This archaeological excavation took place in 5 days in October 2014.

First, to find the site without precise geographical position, it was necessary to realize few polls with a mechanical digger. The bow came first. Quickly, it has appeared that the wreck had suffered greatly since 2002. Many barrel staves of the cargo were mixed with timber of the ship and strewn in the whole hull. This fact caused a slow progression at the beginning of this research. We used a labelling to distinguish elements of the hull, disjoint structures, elements of the cargo and terracotta dishes found in the boat (Figure 5). Before take up all these elements, a photographic coverage was carried out for photogrammetric purposes.

Initially, we hoped to characterize these pieces torn off but it wasn't possible because of their great fragmentation. We finally understood the reasons for the fragmentation of structures. We learned that someone attempted to take a piece of the wreck with a car in 2002, just to take a memory!

During the excavation, the work of the driver of the excavator was threefold. First, he dug a sump in front of the bow, on starboard side, to drain runoff. Then, he created the drains around the wreck and towards the sea to accelerate the evacuation of runoff. We used permanently a water pump to reduce the water level in the sump.

At the end of each day, plastic sheeting were positioned on the wreck and blocked with bags sand and stones. Bags sand was positioned along the sidewalls of the hull to ensure stability. The aim of this device was to limit the return of sand on the excavation area and protect the structures. The next day, after the seawater left the site we quickly removed sand brought by the action of the night tides without damaging the hull. The photographic coverage required for photogrammetric restitution was also made that day for 30 to 40 minutes.

Finally at the end of the day, the excavator made a small mound in the front of the wreck and he created an bypass drain around the wreck to obtain that the tide don't reach the archaeological structures directly.

After the water left the area around the site and after the clean up of the structures of the wreck, the fourth day, was partially dedicated to the implementation of the laser development (two hours while). Day after day, water evacuation, because of the bigger size of the sump hole and all around the wreck, and clean up of the hull took more and more time, more than half working time per day.

The fifth and final day, the team of archaeologists had finalized measurements and observations and made two cross-sections of the wreck, one with batten board and the one without (after cutting it).

For closing the excavation, we protected the site with geotextile and numerous bag of sand.

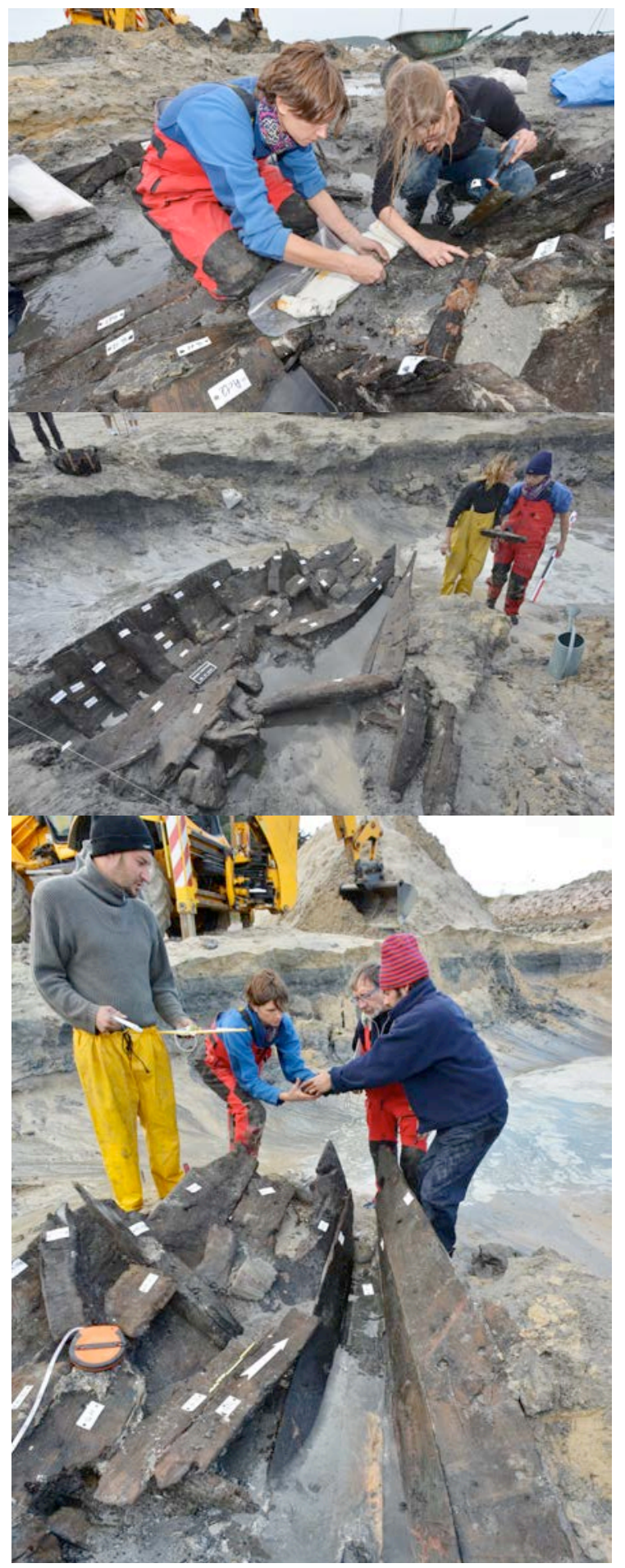

Figure 5. Excavation of the wreck (F.Osada/Drassm) 


\subsection{Photogrammetry scan}

Photogrammetry is based on photographs to reconstruct the shape, dimensions and position of the photographed object. A photographer performed the photogrammetry scan during the last day of the in-situ study. The scan was constituted of 354 pictures, covering a circular view of the wreck.

We used the software Agisoft Photoscan to compute an alignment constituted of 115140 points (Figure 6).

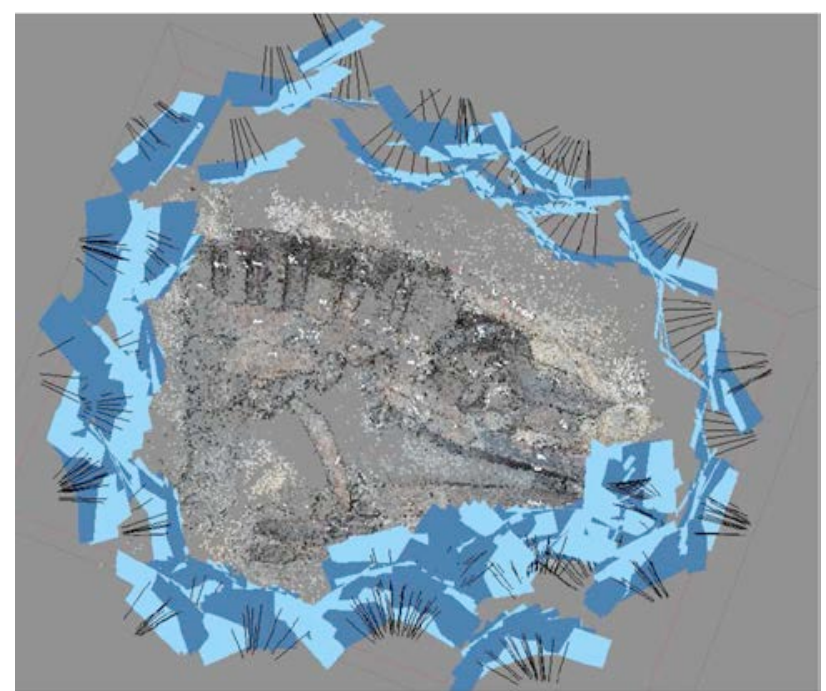

Figure 6. Photos alignment

Then we generated a dense point cloud of 30047845 points (Figure 7), and a 3D model (Figure 8) with 61931072 faces.

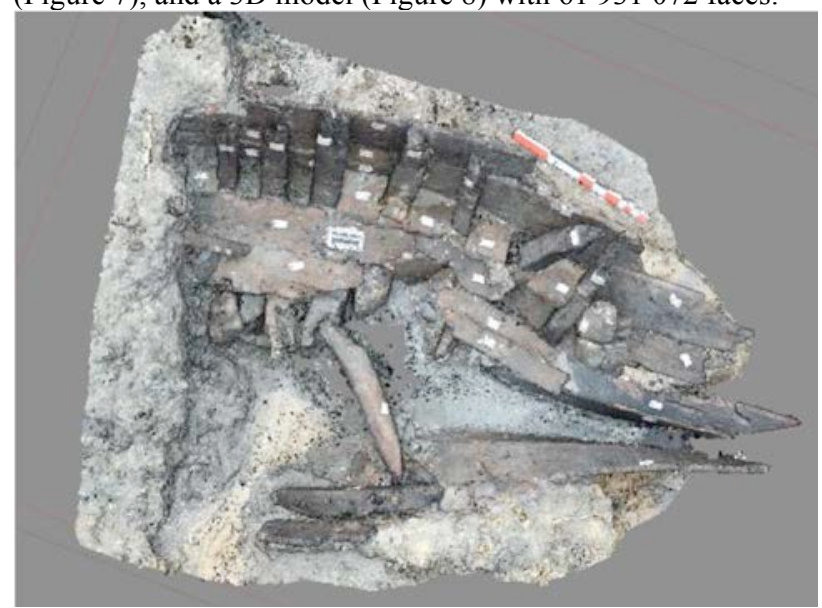

Figure 7. Dense point cloud

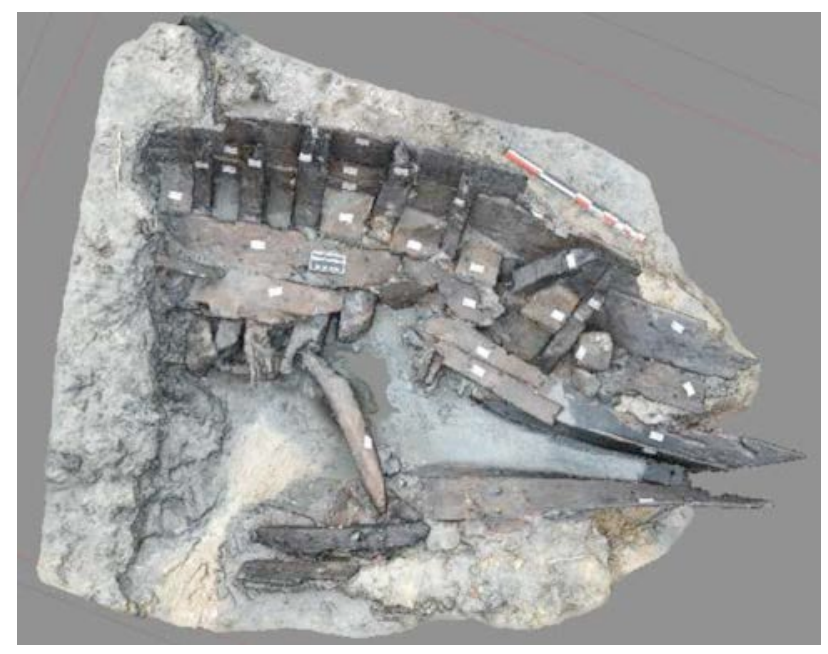

Figure 8. Textured 3D mesh

The model is finally georeferenced with respect to a target positioned on the ground (Figure 9).

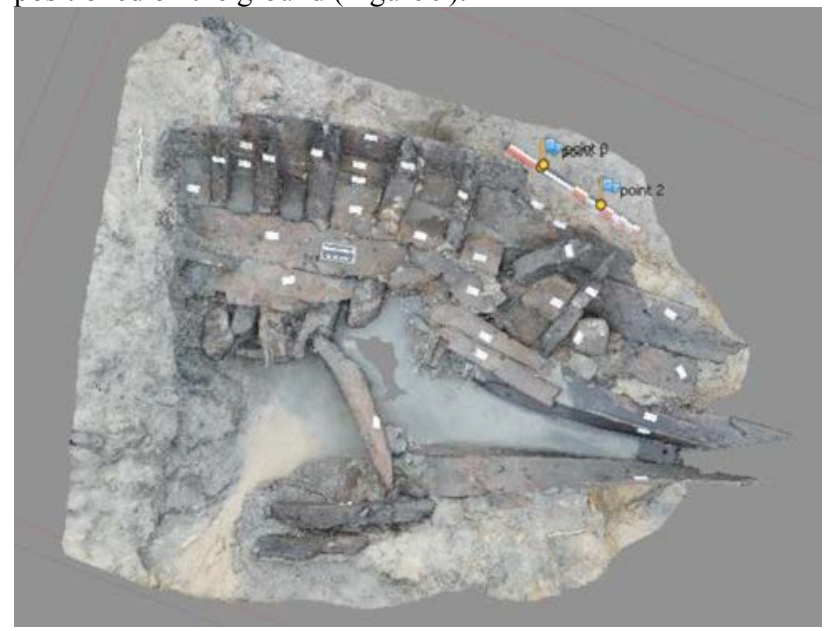

Fig. 9. Geo-referenced mesh

A lighter 3D model was produced $(1,000,000$ faces), from the geo-referenced model, for mediation purposes and collaboration with the virtual reality team. All the 3D models were provided in PLY format, with an associated texture. Vertexes were also colourized, allowing a visualisation without texture.

\subsection{Laser scan}

The laser scanning is based on the principle of the transceiver of a laser light beam on the object. It can acquire several thousand points per second. The alignment of the data of the different stations is based on fixed targets recognised by the laser scanner software. We used four targets during the acquisition.

The scan was conducted by a 3D engineer from West Digital Conservatory of Archaeological Heritage project (Barreau et al., 2013) using a laser scanner FARO Focus 3D X 330 (Figure 10). This scanner was designed specifically for outdoors applications but is quite sensitive to rain and wind that inevitably occur in this region. For all these reasons, it was decided to do short stations but multiplied up in order to secure the cover of the wreck while trying to get the best resolution possible. 

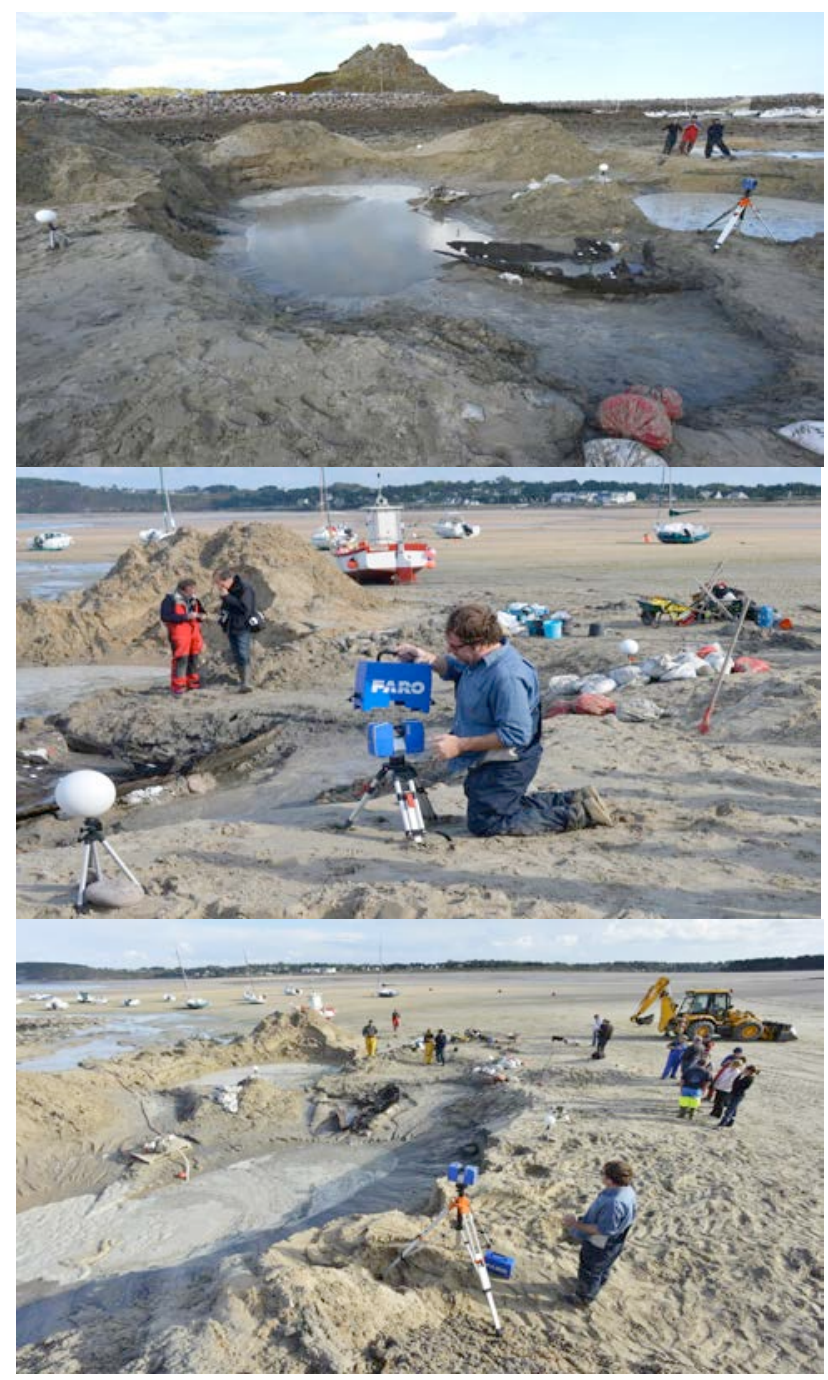

Figure 10. Wreck digitization with laser scanner (F.Osada/Drassm)

Seven stations were performed in 62 minutes, between $3: 13 \mathrm{pm}$ and $4: 15 \mathrm{pm}$. The seven positions of the laser scanner are presented in purple in Figure 11, and the positions of the four laser targets, in green.

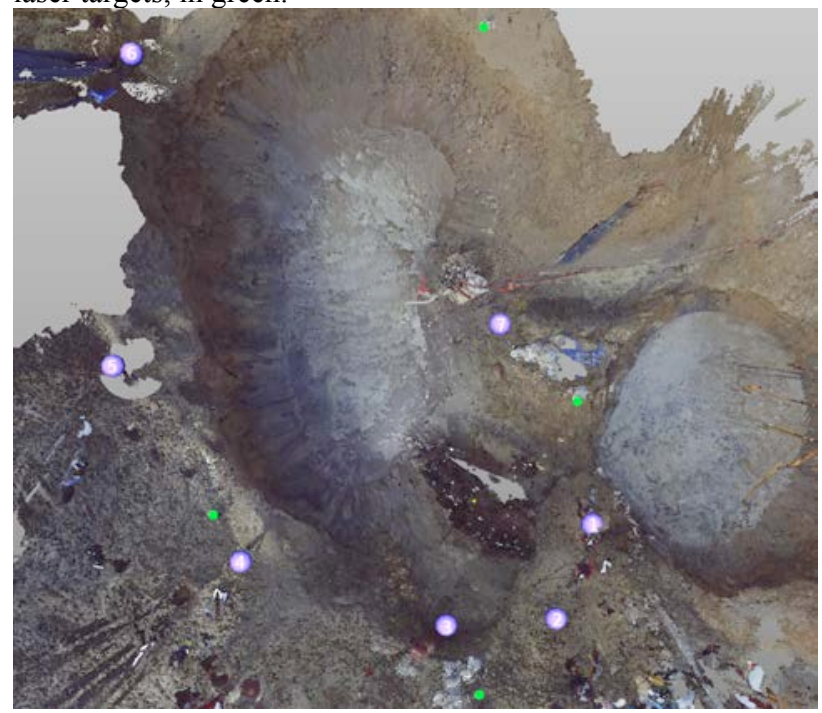

Figure 11. Laser scanner and targets positions
After assembling the 7 stations with the 3D scanned data processing software SCENE, a cloud of 4428295 points was obtained (Figure 12).

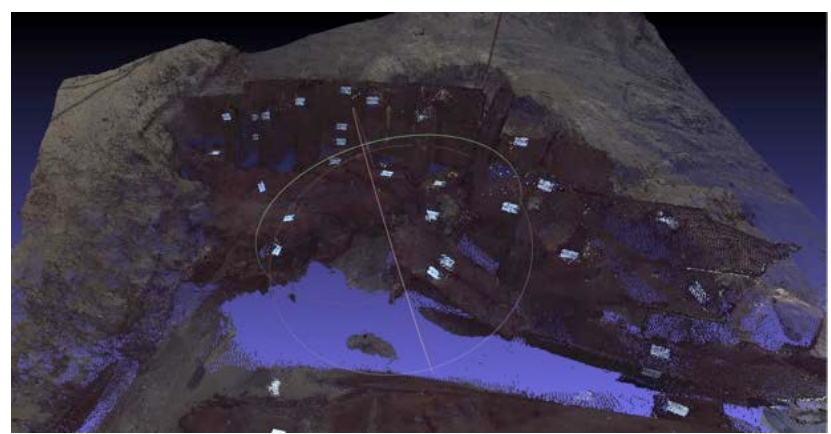

Figure 12. Point cloud

A graphic designer isolated the wreck to get a point cloud of 1252371 points, which represents about $28 \%$ of the initial cloud. A mesh was finally generated with screened Poisson surface reconstruction tool using CloudCompare software (Figure 13)

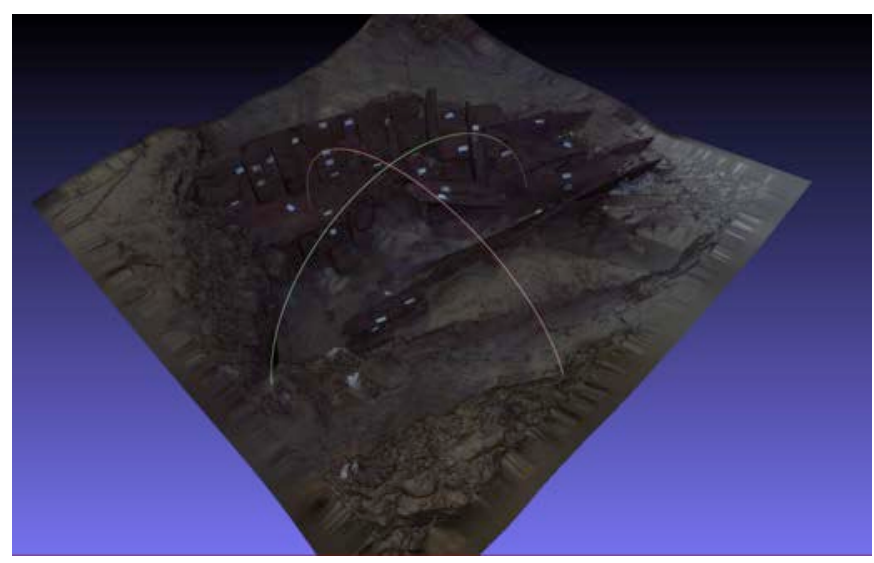

Figure 13. Mesh generated from laser scan

\subsection{Immersive virtual reality implementation}

The goal of the immersive virtual reality implementation was to provide a 1:1 reconstitution of the digitized wreck, with a simple and intuitive navigation mode.

We chose to propose three switchable and co-localised views of the wreck, the point cloud obtained from laser scan, the textured mesh computed from this point cloud, and the textured mesh obtained with photogrammetry. The meshes of the wreck were integrated in a natural reproduction of the environment ambiance, with a skybox coherent with the local climate, a sand ground, and water simulation in the holes around the wreck.

The virtual environment was implemented using was implemented using Unity3D game engine and its virtual reality plugin MiddleVR, and deployed in the Immersia platform (Figure 14). This platform of the IRISA/Inria computer science laboratory is a large virtual reality facility dedicated to realtime, multimodal (vision, sound, haptic, BCI) and immersive interaction. Images are rendered on four glass screens: a front one, two sides and a ground. Dimensions are $9.6 \mathrm{~m}$ wide, $2.9 \mathrm{~m}$ deep and $3.1 \mathrm{~m}$ high. Over 20 millions pixels are displayed. The visual reproduction system combines thirteen HD projectors. A tracking system, composed of 16 infrared cameras, enables real 
objects to be tracked within the scene. Images are recomputed as the user moves to fit his point of view, together with highresolution rendering, active stereoscopy and homogeneous colouring, thereby delivering a visually realistic experience. The Immersia platform has a specific activity in 3D archaeology (Gaugne et al. 2014), in relation with the West Digital Conservatory of Archaeological Heritage project (Barreau et al., 2013).

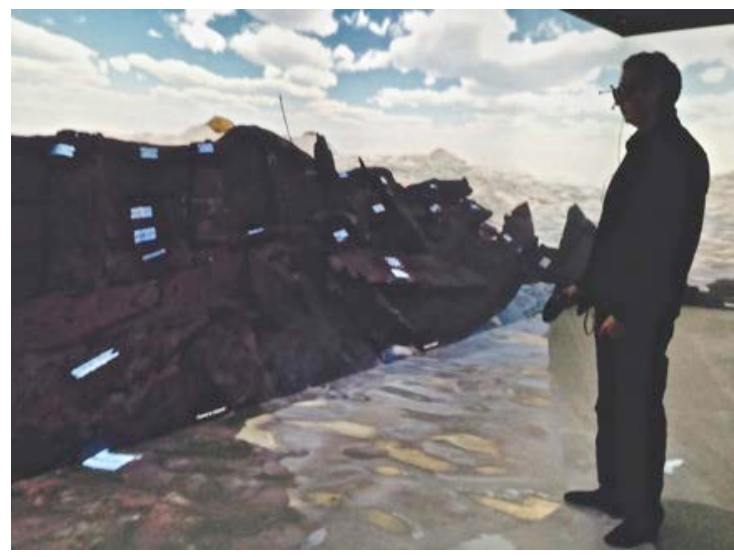

Figure 14. Immersive 1:1 view of the textured mesh in Immersia

In order to display the point cloud (Figure 15), we used an OpenGL rendering in Unity, and a home made parser to open the binary PLY full point cloud (4428295 points) and recover the xyz coordinates and rgb colours. The parser transforms the point cloud into 68 Unity meshes of 65534 and one of 37517 points.

The transformation is performed once, offline, and only Unity meshes are loaded in memory at runtime, to get better performances. It is then possible to interact in Unity with the point cloud as if it was a standard mesh (moves, rendering preview, shaders parameters).

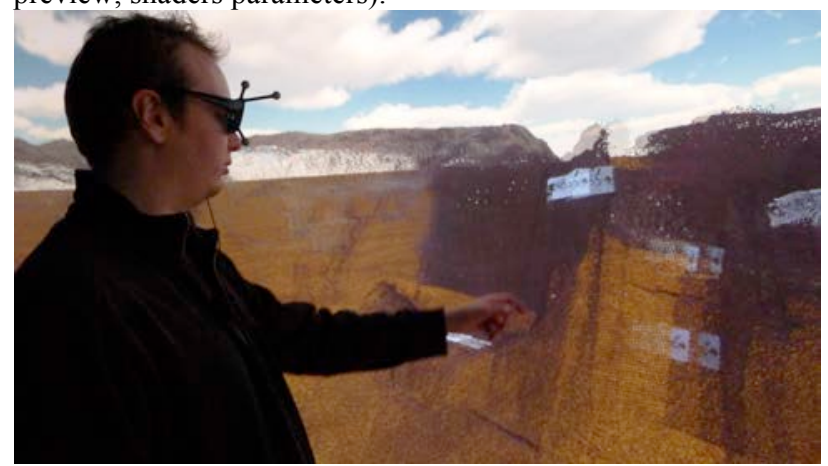

Figure 15. Immersive 1:1 view of the point cloud

Interaction is provided through an ART Flystick 2 device (Figure 16), which includes one joystick, five buttons and one trigger and whose position and orientation are tracked by the infrared cameras. The user navigates in a flying mode, he moves with the joystick, according to the direction pointed by the Flystick. He can also naturally walk on the $30 \mathrm{~m}^{2}$ of the floor screen. One button is used to switch between the three renderings of the wreck.

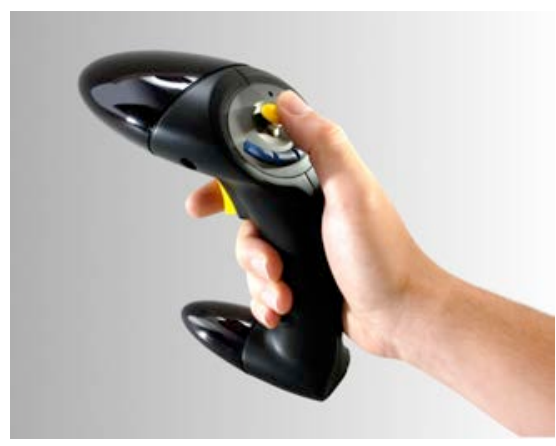

Figure 16. The Flystick 2 device

\section{DISCUSSION}

The process defined in this work allowed to perform an efficient study of a wreck situated in the inter-tidal area. In particular, the digitization techniques used in-situ produced valuable data, in a short time. These data constitute a long-term digital recording of the site that can be exploited a posteriori during the postexcavation period and without requiring a new operation on the wreck.

Tools associated to $3 \mathrm{D}$ data were used to generate $2 \mathrm{D}$ data useful for the reporting of the study. For example, laser data was used to generate ortho-photos of the wreck (Figure 17) and cutting plans of the site (Figure 18). Photogrammetry results were used to generate a general planimetry of the wreck (Figure 19).

For photogrammetry and restitution with photoscan, the margin of error is $+/-3 \mathrm{~mm}$. Even with the best archaeological drawing in the field with traditional tools (plumb line and decametre) the error definitely couldn't be more precise.

We encountered problem with the seawater and runoff always present in the wreck during the work... and nor the laser, neither the photogrammetric coverage succeeded in penetrating the water to capture data. Only the manual work could overcome this pitfall.

The use of traditional archaeological techniques offers a precise comparison between the results obtained. This operation is continued currently.

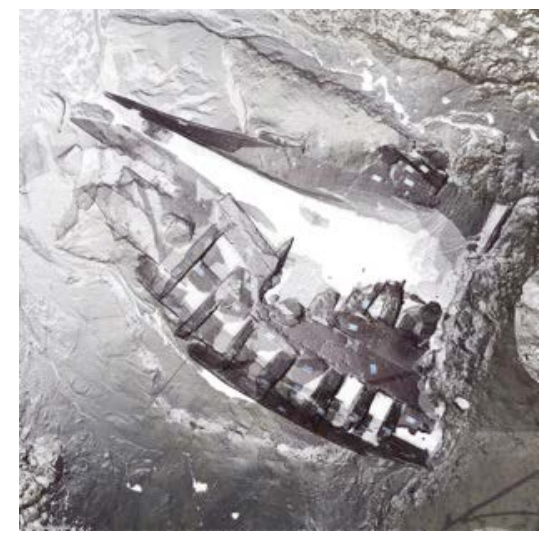

Figure 17. Ortho-photo of the wreck 


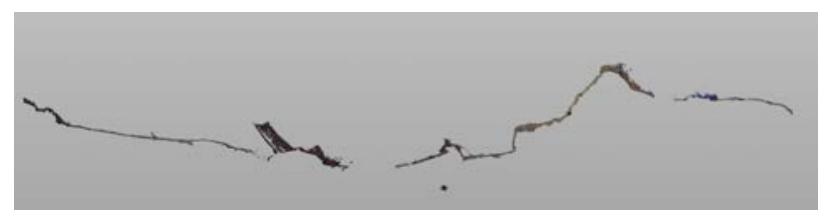

Figure 18. Generated cutting plan

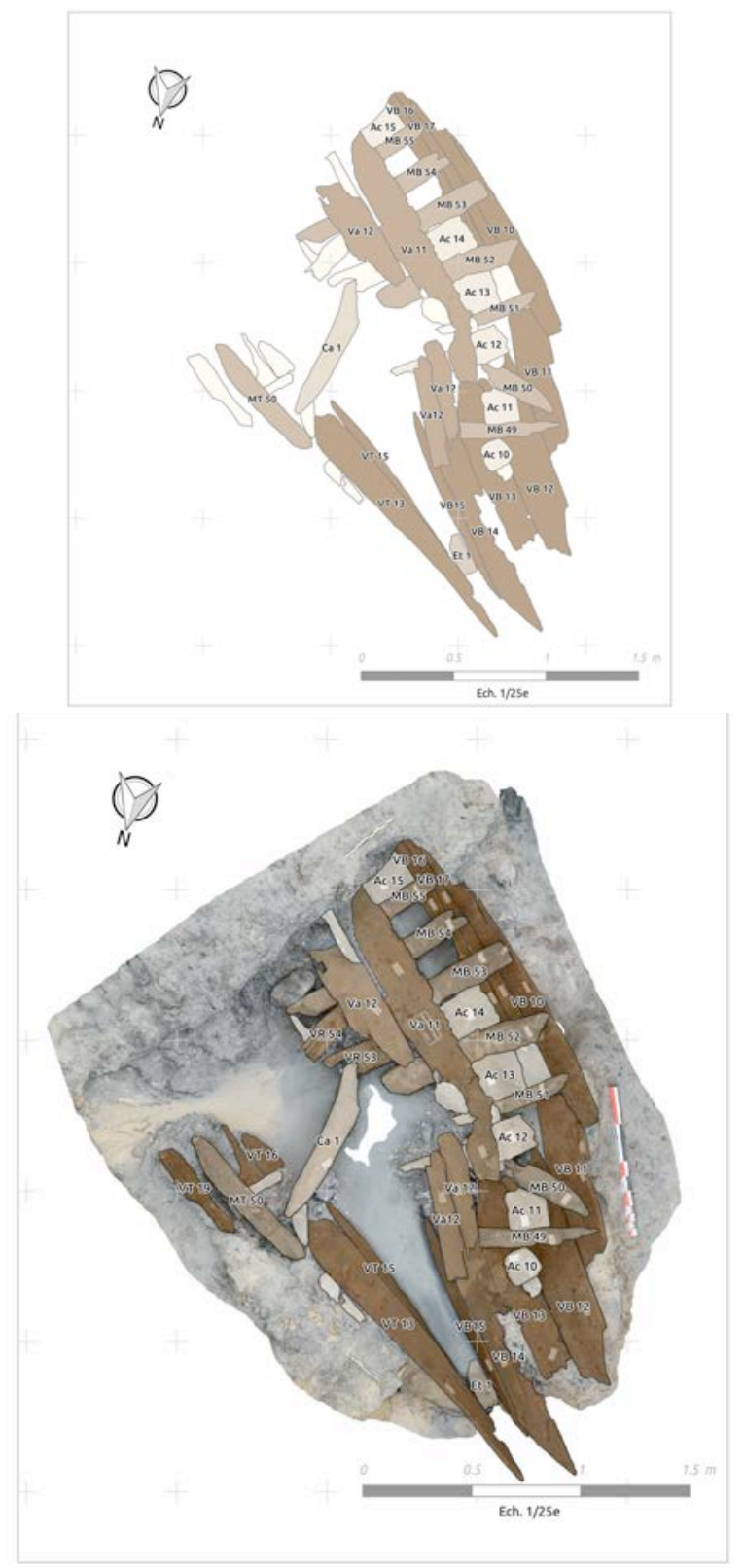

Figure 19. General planimetry (D.Dégez/Drassm)

Without going into the "lidar vs photogrammetry" classic debate, it appears that the acquisition by photogrammetry allowed a better density and a very good quality texture for the 3D model. Simultaneously and for later potential applications, laser scanning has the following advantages: light independence, thin vegetation / algae penetration, minimum human dependence, fast acquisition and processing, potential high accuracy. With regard to this latter issue if there is time to scan, long stations enable a accuracy up to $\pm 2 \mathrm{~mm}$.

\section{CONCLUSION}

The excavation of Erquy 's wreck is a site laboratory and allowed us to use and compare the results of two technics of digitalization. Those technics are now well known and offer good results for many scientific applications.

In the inter-tidal context, heritage is really fragile and the risk of destruction is real (storms, erosion, coastal development...). The traditional methods are no longer efficient.

A virtual reality tool that would be interesting to develop could generate cutting planes directly in virtual reality. Archaeologist immersed in 1:1 reconstitution with an interaction device could himself manipulate his $3 \mathrm{D}$ virtual cutting plane, position it accurately and automatically generate the $2 \mathrm{D}$ cutting planes that he needs for his reports.

Virtual reality implementation allow archaeologists to came back to a 1:1 reconstitution of the site and continue their analysis work in an virtual environment without new excavation. This immersive 1:1 reconstitution enables a sensory-motor experience that is out of reach with a simple view of the $3 \mathrm{D}$ data on a screen. This is particularly interesting to offer the possibility of a realistic exploration of the site for archaeologists who did not have the possibility to view the wreck when it was excavated. Virtual reality reconstitutions also constitute an interesting tool for cultural heritage valorisation and scientific mediation.

From data acquisition to exploitation of the results for all public (scientific community and general public), many aspects of new technologies have been studied. Our results demonstrate the necessity to continue this perfectible experience the next year because of the partial excavation of the boat. The partial results require continuing our comparative tests until the merger of the models of the bow (excavate in 2014) and those of the stern (scheduled for 2015) of Erquy's boat. A new aspect will be performed in 2015, to test on the site the realization of an electric prospection on the sand, on the top of the wreck to determine a singular signature. This study will be interesting to find some wrecks hidden under the sediments without geographical position. The research continues also for dendrochronological aspects for fine tune of the dating of this modern ship.

Already, the study of other wrecks are envisaged by the Drassm in 2016, after Erquy's study, to determine the best protocol adapted to emergency situations and also for annual excavation's projects.

\section{ACKNOWLEDGEMENTS}

This work is supported by the French Ministry of Culture and Communication, the French Ministry of Higher Education and Research, the regional council of Région Bretagne, and UEB. The authors wish to warmly thank the all team of the excavation project especially Eric Rieth and Catherine Lavier (CNRS). Thanks also to Stéphane Jupin et Aurélie Guesdon (Services 3D) for their precious help for the post project treatment based on data restitution.

\section{REFERENCES}

Anderson et al. 2012. Anderson, R., and McAllister, M., Koombana Bay foreshore maritime archaeological survey and excavations, Report - Department of Maritime Archaeology, Western Australian Museum No. 286, May 2012. 
Barreau et al., 2013. Barreau, J-B., Gaugne, R., Bernard, Y., Le Cloirec, G., and Gouranton, V., 2013. The West Digital Conservatory of Archaeological Heritage project, in Proc. Of Digital Heritage International Congress 2013, Vol. 1, pp 547554 .

Barreau et al., 2014, Barreau, J-B., Bernard, Y., Petit, Q., Beuchet, L., Petit, E., Platen, V., Gaugne, R., Le Rumeur, J., and Gouranton, V., Combination of 3D Scanning, Modeling and Analyzing Methods around the Castle of Coatfrec Reconstitution, in Proc. of 5th International Euro-Mediterranean Conference - EuroMed 2014.

Bell et al. 2000. Bell, M.G., Caseldine, A.E. and Neumann, H. 2000 Prehistoric Intertidal Archaeology in the Welsh Severn Estuary, CBA Research Report 120: York.

Buchet et al. 2004. Buchet, C., Meyer, J., Poussou J.-P. La puissance maritime, PUPS, Paris, 2004, 651 p.

Draper 2007. Draper, S., A Survey and study of the background of the wreck of the Steam Trawler 'Sheraton' lying in the intertidal zone off Hunstanton, North Norfolk, Survey Report in Part Fulfilment of the Requirements of the NAS Part II Intermediate Certificate in Foreshore and Underwater Archaeology, 2007

Gaugne et al. 2014. Gaugne, R., Gouranton, V., Dumont, G., Chauffaut, A., and Arnaldi, B., Immersia, an open immersive infrastructure: doing archaeology in virtual reality, in Archeologia e Calcolatori, supplemento 5 (2014) 1-10).

Le Gall and Blouin 1994. Le Gall, J.-P., Blouin R., Le port d'Erquy au temps des carrières, Chasse-Marée n82, Tours, p. 32-41, 1994.

Le Bouedec 2008. Le Bouedec G., Le cabotage sur la façade atlantique française (XVI- XIXè siècles), Revue d'Histoire Maritime nº 8 , PUPS, Paris, p. 9-37, Feb, 2008.

L'Hour and Veyrat 2002. L'Hour M., Veyrat E. L'épave des Hôpitaux, plage des Montiers à Erquy, 2002, Bilan scientifique du Drassm, Marseille p. 26-29.

Matsumoto and Ono 2009. Matsumoto, K., and Ono, I. 2009, Improvements of archaeological excavation efficiency using $3 D$ photography and Total Stations. In: 22nd CIPA Symposium, October 11-15, 2009, Kyoto, Japan.

Nayling and Jones 2009. Nayling, N., and Jones, T., ThreeDimensional Recording and Hull Form Modelling of the Newport (Wales) Medieval Ship, Proceedings of the 12th Symposium on Boat and Ship Archaeology, Istanbul 2009.

Prins and Adams 2012. Prins, A., and Adams, M.J., Practical Uses for Photogrammetry on Archaeological Excavations, JVRP White Papers in Archaeological Technology, December, 2012.

Rieth 2014. Rieth E., Archéologie des épaves de l'estran: l'exemple de la fouille de l'épave des Hôpitaux (Côtes-d'Armor), Neptunia n²76, Paris, 2014, p. 60-62.

Vozikis et al. 2004. Vozikis, G., Haring, A., Vozikis E., and Kraus K., Laser Scanning: A New Method for Recording and Documentation in Archaeology, in Workshop - Archaeological Surveys WSA1 Recording Methods, Athens, Greece, May 2227, 2004.
Watson 2005. Watson, C. Seahenge: An Archaeological Conundrum. Swindon: English Heritage, 2005. 